\title{
Stabilization of HIF-1a alleviates osteoarthritis via enhancing mitophagy
}

\author{
Sunli $\mathrm{Hu}^{1,2,3}$, Chunwu Zhang ${ }^{4}$, Libin $\mathrm{Ni}^{1,3}$, Chongan Huang ${ }^{1,3}$, Dingwen Chen ${ }^{2,3}$, Keqing Shi ${ }^{4}$, Haiming Jin ${ }^{1}$, \\ Kairui Zhang ${ }^{1,2,3}$, Yao Li ${ }^{1,2,3}$, Ling Xie ${ }^{2}$, Mingqiao Fang ${ }^{1,2,3}$, Guangheng Xiang ${ }^{1,2,3}$, Xiangyang Wang ${ }^{1}$ and Jian Xiao $\mathbb{D}^{1,2}$
}

\begin{abstract}
Mitochondrial dysfunction leads to osteoarthritis (OA) and disc degeneration. Hypoxia inducible factor-1a (HIF-1a) mediated mitophagy has a protective role in several diseases. However, the underlying mechanism of HIF-1a mediated mitophagy in OA remains largely unknown. This current study was performed to determine the effect of HIF-1a mediated mitophagy on OA. Therefore, X-ray and tissue staining including HE staining, safranin O-fast green (S-O) and Alcian Blue were used to assess imageology and histomorphology differences of mouse knee joint. Transcriptional analysis was used to find the possible targets in osteoarthritis. Western blot analysis, RT-qPCR and immunofluorescence staining were used to detect the changes in gene and protein levels in the vitro experiment. The expression of HIF-1a was increased in human and mouse OA cartilage. HIF-1a knockdown by siRNA further impair the hypoxia-induced mitochondrial dysfunction; In contrast, HIF-1a mediated protective role was reinforced by prolylhydroxylase (PHD) inhibitor dimethyloxalylglycine (DMOG). In addition, HIF-1a stabilization could alleviate apoptosis and senescence via mitophagy in chondrocytes under hypoxia condition, which could also ameliorate surgery-induced cartilage degradation in mice OA model. In conclusion, HIF-1a mediated mitophagy could alleviate $O A$, which may serve as a promising strategy for $O A$ treatment.
\end{abstract}

\section{Introduction}

Osteoarthritis (OA) is a leading cause of disabling disease worldwide ${ }^{1}$. It imposes a huge burden on the family affected, social welfare institutions and the social economic cost ${ }^{2}$. The incidence appears to be increased in the past decade. Especially, the incidence of osteoarthritis increased sharply in the elderly people ${ }^{3}$. Clinically, knee joint is the most common site of arthritis, followed by the hand and hip ${ }^{4}$. However, pain is the most common clinical symptom of osteoarthritis ${ }^{5}$. When noninvasive and conservative treatment fails to achieve relieving symptom, surgery is the only last choice for patients ${ }^{6}$.

\footnotetext{
Correspondence: Xiangyang Wang (xiangyangwang@wmu.edu.cn) or Jian Xiao (xfxj2000@126.com)

'Department of Orthopaedics, The Second Affiliated Hospital and Yuying Children's Hospital of Wenzhou Medical University, Wenzhou, Zhejiang, China ${ }^{2}$ Molecular Pharmacology Research Center, School of Pharmaceutical Science, Wenzhou Medical University, Wenzhou, Zhejiang, China Full list of author information is available at the end of the article Edited by G.M. Fimia
}

Mitochondria is one of the most complex and important organelles found in eukaryotic cells and carries out several biochemical processes, maintains energy production through adenosine triphosphate (ATP) generation ${ }^{7}$. Mitochondrial dysfunction is thought to be associated with extracellular matrix metabolism, apoptosis, aging and a range of pathological processes, including arthritis and disc degeneration ${ }^{8,9}$.

Autophagy is an important part of cellular process, which has dual role in chondrocyte fate. Studies have revealed the cytoprotective role of autophagy in OA development, while it also can lead to autophagic cell death ${ }^{10-12}$. Several researchers strengthened knowledge of the protective role of autophagy against both chondrocyte apoptosis and OA development. Autophagy-related proteins such as Beclin-1and LC3, were markedly increased in human chondrocytes and associated with increased apoptosis ${ }^{10}$. In an OA mouse model, Carames et al. showed that OA development is accompanied by a loss of

\section{(c) The Author(s) 2020}

(c) (i) Open Access This article is licensed under a Creative Commons Attribution 4.0 International License, which permits use, sharing, adaptation, distribution and reproduction cc) in any medium or format, as long as you give appropriate credit to the original author(s) and the source, provide a link to the Creative Commons license, and indicate if changes were made. The images or other third party material in this article are included in the article's Creative Commons license, unless indicated otherwise in a credit line to the material. If material is not included in the article's Creative Commons license and your intended use is not permitted by statutory regulation or exceeds the permitted use, you will need to obtain permission directly from the copyright holder. To view a copy of this license, visit http://creativecommons.org/licenses/by/4.0/. 
key regulators of autophagy and an increase of apoptosis, as determined by PARP cleavage ${ }^{11}$. Administration of rapamycin alleviated the severity of experimental $\mathrm{OA}^{13}$ while silencing of beclin-1 resulted in enhanced chondrocyte death. Mitophagy is a special form of autophagy that maintains mitochondrial homeostasis via eliminating impaired organelles, unwanted protein and reducing cellular stress caused by harmful stimulus ${ }^{14}$. Basal levels of mitophagy maintain cellular homeostasis and protect cells. During several cellular stress including high glucose, oxidative stress and low-oxygen conditions, mitophagy can be stimulated whereby up-regulated mitophagy level can promote cell survival by removing damaged mitochondria ${ }^{15}$. Mitophagy has been associated with mitochondrial dysfunction and apoptosis in the pathological process of several diseases, including heart disease ${ }^{16}$, neurodegenerative diseases ${ }^{17}$ and kidney diseases ${ }^{18}$.

Hypoxia inducible factor-1 (HIF-1) is a heterodimer composed of oxygen-sensitive HIF- $1 \alpha$ and constitutively expressed HIF-1 $\beta$ subunits, regulates adaptive responses to hypoxia conditions ${ }^{19-21}$. Under normal conditions, the HIF- $1 \alpha$ subunit is rapidly degraded by prolyl-4hydroxylases (prolyl hydroxylation domain protein, PHD), a major biodegradable small molecule of HIF- $1 \alpha$. PHD is less active during hypoxia or ischemia ${ }^{22,23}$, which leads to inhibiting the HIF- $1 \alpha$ degradation and causing the HIF- $1 \alpha$ to transfer into the nucleus whereas it recruits HIF- $1 \beta$, inducing the expression of specific target genes, such as BNIP3, an essential molecule for mitophagy ${ }^{23,24}$. In addition, the damaged mitochondria clearance by mitophagy can prevent from ROS synthesis and apoptotic cell death ${ }^{25}$.

HIF- $1 \alpha$ plays a significant role in chondrocyte survi$\mathrm{val}^{26}$. HIF-1 $\alpha$ was detected in the nuclear extracts of chondrocytes which were isolated from normal or OA cartilages and cultivated under normoxic condition. HIF$1 \alpha$ conditional $\mathrm{KO}$ led to massive chondrocyte cell death in the growth plate ${ }^{27}$. Bohensky et al. suggested that prior to their apoptotic cell death, promoting chondrocytes survival in an autophagic state, which was stimulated by HIF- $1 \alpha^{28}$. The mechanisms of HIF- $1 \alpha$ induced autophagy contains modulation of beclin-1/Bcl-2 complex $^{28}$ and inhibition of $\mathrm{mTOR}^{29}$. Recently, Chen et al. demonstrated the potential role of Bcl- 2 modulation in HIF- $1 \alpha$-mediated autophagy ${ }^{30}$. HIF- $1 \alpha /$ HIF- $2 \alpha$ imbalance is a main regulator of chondrocyte survival/death. HIF-1 $\alpha$ expression is decreased and HIF- $2 \alpha$ is increased, skewing the imbalance resulting in autophagy and apoptosis. Unlike HIF- $1 \alpha$, HIF- $2 \alpha$ is a negative regulator of the autophagy. HIF- $2 \alpha$-silenced chondrocytes exhibited high elevation of lysosomal activity, inhibition of mTOR expression and the presence of autophagosomes. Previous studies failed to elaborate the relationship between HIF-1 $\alpha$ and mitophagy in osteoarthritis. Downstream signaling mechanisms of mitophagy in osteoarthritis are remained unclear until now. In our present study, we identified that hypoxia induced mitophagy could protect chondrocyte from apoptosis, and senescence. HIF-1 $\alpha$ overexpression could enhance mitophagy in vitro experiments. DMOG mediated HIF- $1 \alpha$ up-regulation inhibits the DMM-induced cartilage degradation. In summary, HIF-1 $\alpha$ may become a potential target for treating osteoarthritis.

\section{Results \\ HIF-1a was increased in human and mouse knee articular cartilage}

Previous studies show that mitophagy induces chondrocytes degeneration, which indicated that HIF- $1 \alpha$ is related to the OA development. To confirm the relationship between $\mathrm{OA}$ and $\mathrm{HiF}-1 \alpha$, western-blot analysis was performed in the chondrocytes in human knee cartilage tissue of normal and OA patients. X-ray images of normal and OA patient were displayed in Fig. 1a. Therefore, our significant results showed that HIF-1 $\alpha$ expression level was enhanced in human $\mathrm{OA}$ group compared to the healthy control (Fig. 1b, c). Besides, to verify the HIF- $1 \alpha$ changes in mouse chondrocytes in vitro, the mouse chondrocytes were cultured in incubators with different oxygen concentrations $\left(1,3,5 \% \mathrm{pO}_{2}\right)$ for $24 \mathrm{~h}$ and hypoxia condition $\left(1 \% \mathrm{pO}_{2}\right)$ for $6,12,24 \mathrm{~h}$ (Fig. 1d-g). Then, the HIF- $1 \alpha$ protein expression levels were measured in each group by western-blot analysis. We found that the HIF-1 $\alpha$ protein expression levels were considerably elevated in the time-dependent manner and under hypoxia. The HIF- $1 \alpha$ level expression levels were enhanced in chondrocytes cultured in hypoxia condition $\left(1 \% \mathrm{pO}_{2}\right)$ for $24 \mathrm{~h}$. Thus, we selected this condition for the subsequent experiments. To elucidate further biological manners of chondrocytes in hypoxia condition, RNA sequencing was conducted to explore the transcription profiles of mouse chondrocytes treated with hypoxia stimulation for $24 \mathrm{~h}$ or not. Total 1202 differently expressed genes were identified (including up and down genes) between Control vs Hypoxia group (Fig. 1h, i). According to the functional analysis of dysregulated mRNAs, we found that metabolic pathways, apoptosis, autophagy and cellular senescence which were tightly associated with osteoarthritis (Fig. 1j). Interestingly, BNIP3 interacts with Beclin inducing mitophagy was markedly up-regulated and enriched (Fig. 1k). Thus, these substantial data suggested that HIF-1 $\alpha$ was augmented in OA and hypoxia condition and the increased HIF- $1 \alpha$ expression regulated mitophagy, apoptosis and senescence makers.

\section{Hypoxia stimulated mitochondrial ROS generation, apoptosis, and autophagy in chondrocytes}

Fluorescence staining and western-blot were performed to detect mitochondrial ROS generation, apoptosis and 
A

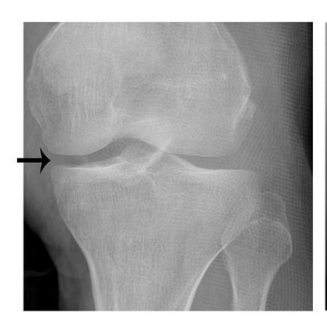

Relative Normal

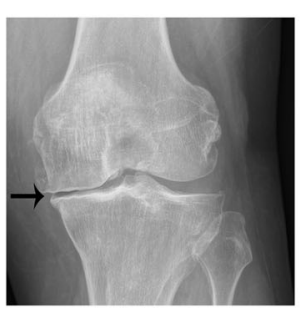

OA

D

B
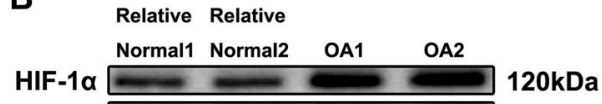

GAPDH

$36 \mathrm{kDa}$

C

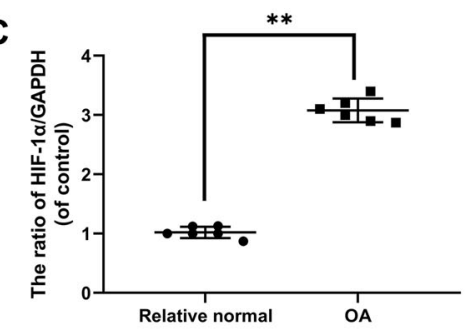

E

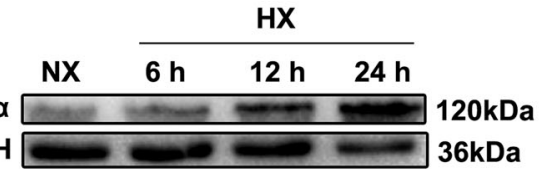

F

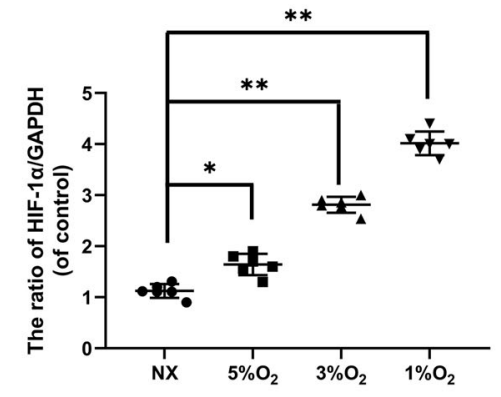

H

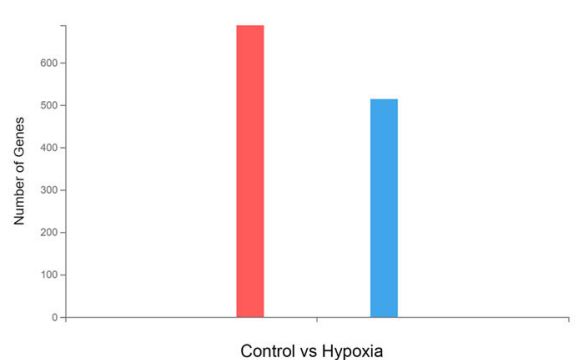

J

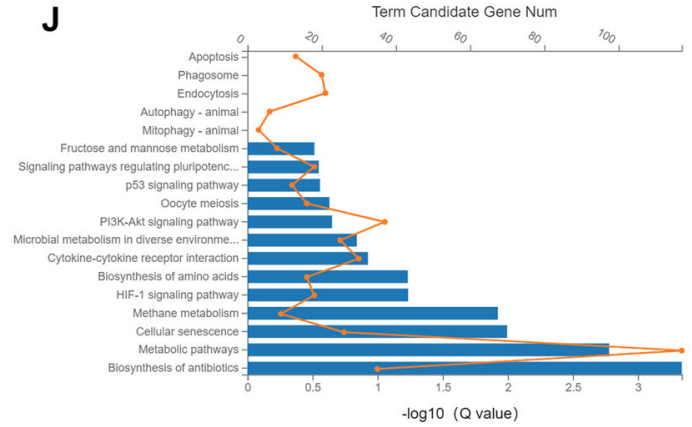

G
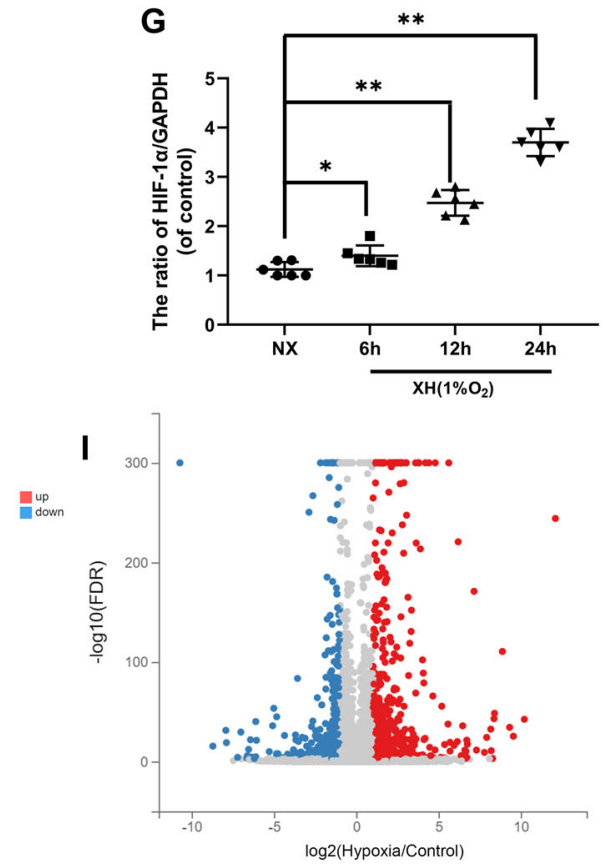

K

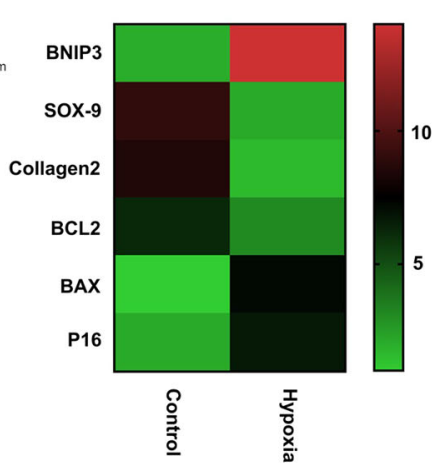

Fig. 1 (See legend on next page.) 
(see figure on previous page)

Fig. 1 HIF-1a is increased in human and mouse knee articular cartilage. a Representative $X$ ray images of normal and osteoarthritis patients. b The protein expression of HIF-1a in human chondrocytes derived from normal and osteoarthritis patients. c Quantification of HIF-1a by immunoblots. $\mathbf{d}$, e The expression of HIF-1a from hypoxia treated mouse chondrocytes was analyzed by western blot. $\mathbf{f}, \mathbf{g}$ Quantification of HIF-1a immunoblots. h-k Bioinformatic histogram, Volcano Plot, KEGG and Heatmap analyses of the key molecules and signaling pathways differentially regulated in mouse chondrocytes treated with hypoxia condition $\left(1 \% \mathrm{pO}_{2}\right)$ for $24 \mathrm{~h}$ based on transcriptome analysis. All data represent mean \pm S.D. $(n=6) .{ }^{*} P<0.01,{ }^{*} P<0.05$

autophagy respectively in hypoxia-stimulated chondrocytes. Compared with control group, the Bax, cleavedcaspase3 and Cytochorome $\mathrm{C}$ (Cyto C) protein expression levels were significantly increased in hypoxia stimulated group, while $\mathrm{Bcl}-2$ (the anti-apoptotic protein) expression level was significantly decreased (Fig. 2a, b). Moreover, western blotting data showed that hypoxia not only led to apoptosis but also induced autophagy (Fig. 2a, b). The LC3-II/I ratio was significantly increased while p62 was elevated in hypoxia-treated chondrocytes, indicating that autophagy flux was impaired. In order to confirm autophagy activation, bafilomycin is an autophagosome degradation inhibitor used to quantize autophagic flux activity. Our remarkable results demonstrated that autophagy was activated (Fig S1). The mitochondrial ROS level was significantly increased in hypoxia condition $\left(1 \% \mathrm{pO}_{2}, 24 \mathrm{~h}\right)$, as well as changes in mitochondrial transmembrane potential, leading to mitochondrial damage (Fig. 2c-e, g). TUNEL assay and Annexin V-FITC revealed that hypoxia could stimulate apoptosis (Fig. 2c, e, g). JC-1 is an ideal fluorescent probe widely used to detect mitochondrial membrane potential (mitochondrial be potential) $\Delta \Psi \mathrm{m}$. The jc-1 transformation from red fluorescence to green was an early apoptosis indicator, suggesting that hypoxia can impair mitochondrial bioenergetics via decreasing $\Delta \Psi \mathrm{M}$ (Fig. 2f).

In summary, these substantial findings indicated that hypoxic environment could cause chondrocytes apoptosis, activate autophagy and enhance mitochondrial ROS.

\section{HIF-1a was associated with hypoxia-induced chondrocytes self-protection}

HIF- $1 \alpha$ expression levels were altered in hypoxiatreated chondrocytes. To further verify whether HIF- $1 \alpha$ played a protective role in hypoxia-induced OA, mitochondrial function, apoptosis and autophagy related indicators were assessed in rat chondrocytes transfected HIF- $1 \alpha$ siRNA (as shown in Fig. 3a, b). HIF- $1 \alpha$ expression levels were significantly inhibited. In addition, the Bax, cleaved-caspase 3 and Cytochrome $\mathrm{C}$ protein expression levels were further increased using HIF- $1 \alpha$ knockdown while $\mathrm{Bcl} 2$ expression levels were significantly augmented (Fig. 3c, d). The results showed that HIF-1 $\alpha$-knockdown further increased hypoxic-induced apoptosis and mitochondrial ROS production as well as decreased $\Delta \Psi_{\mathrm{M}}$ in chondrocytes, demonstrating that decreased HIF- $1 \alpha$ expression level impaired energy production and mitochondrial functions (Fig. 3e, f, i). TUNEL also confirmed that HIF-1 $\alpha$ knockdown promoted hypoxia-induced apoptosis (Fig. 3g, h). Double staining of HIF- $1 \alpha$ and Tom20 (mitochondrial membrane protein marker) showed that si-RNA reduced the HIF-1 $\alpha$ aggregation in mitochondria in hypoxia-stimulated chondrocytes (Fig. 3j). In summary, these significant data indicated that HIF- $1 \alpha$ might play a protective role in chondrocytes survival in oxygen-deficient environment.

\section{DMOG increased HIF-1a expression, protects against hypoxia-induced apoptosis and autophagy flux}

Previous studies have shown that DMOG, a PHD inhibitor is a promising treatment for ischemic hypoxic disease through HIF-1 $\alpha$ dependent mechanism. In our current investigation, we performed whether DMOG could increase chondrocyte survival and the therapeutic effects of HIF- $1 \alpha$ in chondrocytes. Firstly, DMOG was not cytotoxic to chondrocytes at $0,0.1,0.5,1,2,4 \mathrm{~m}$ concentrations, $\mathrm{M}$ after treatment for $24 \mathrm{~h}$ (Fig. $4 \mathrm{a}$ ) by CCK- 8 . Furthermore, under hypoxic conditions, $1 \mathrm{mM}$ and $2 \mathrm{mM}$ DMOG concentrations maintained high cellular chondrocytes viability (Fig. 4b). Chondrocytes treated with the increasing DMOG concentration displayed enhancing HIF- $1 \alpha$ level, suggesting that DMOG could increase the HIF-1 $\alpha$ expression levels in chondrocytes. Similarly, DMOG activated autophagy by increasing LC3II, and the P62 which was significantly reduced under hypoxic condition, indicating that blocked autophagy flux was recovered. Also, the pro-apoptotic proteins (Cleavedcaspase 3 and $\mathrm{Bax}$ ) and mitochondria related protein cytochromes C (Cyto C) expression levels were significantly reduced with DMOG treatment while antiapoptotic protein (Bcl-2) was markedly increased in DMOG group in a dose dependent manner (Fig. 4c-f). Fluorescence staining for HIF- $1 \alpha$ in the chondrocytes showed that HIF- $1 \alpha$ intensity was significantly increased after DMOG treatment (Fig. 4g, h). Thus, these significant results indicated that DMOG could increase HIF- $1 \alpha$ expression, activate autophagy and suppress apoptosis in hypoxia stimulated chondrocytes. 
A

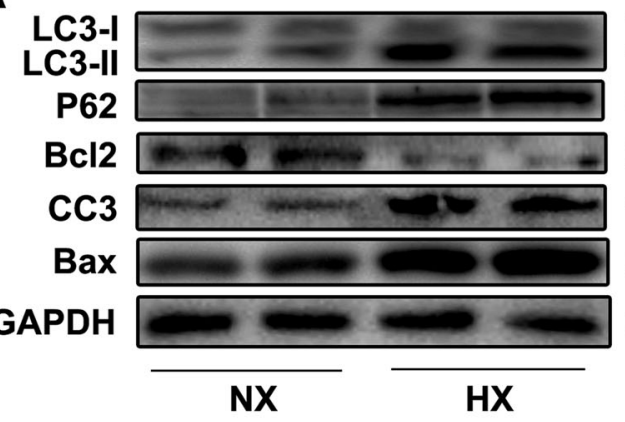

$16 \mathrm{kDa}$ $14 \mathrm{kDa}$

$62 \mathrm{kDa}$

$27 \mathrm{kDa}$

$17 \mathrm{kDa}$

$20 \mathrm{kDa}$

$36 \mathrm{kDa}$

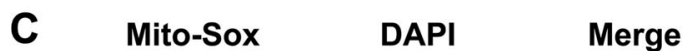

B

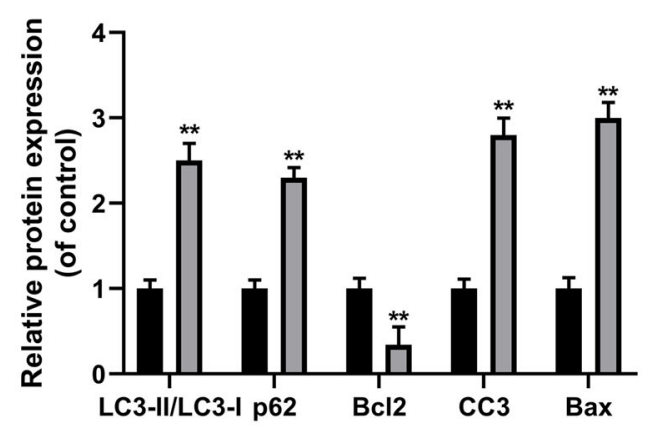

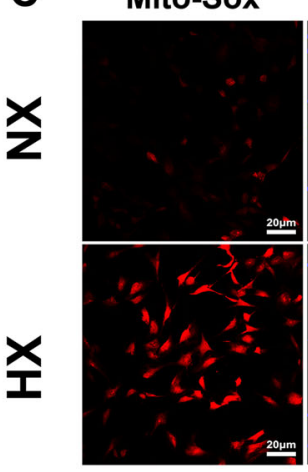

D

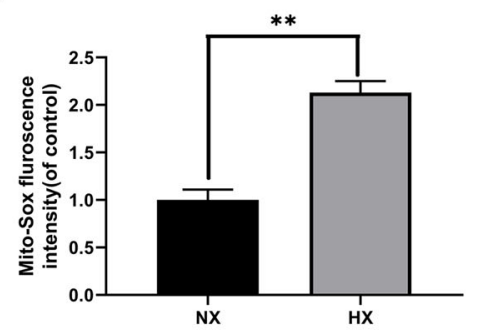

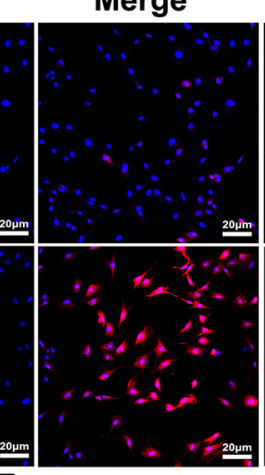

E

TUNEL

DAPI

Merge

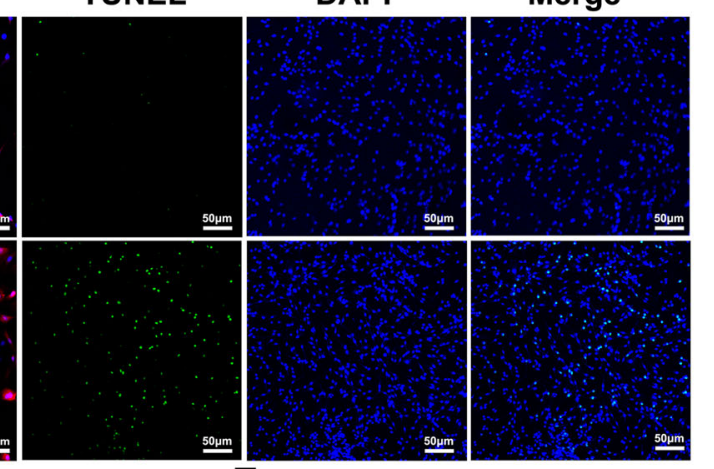

$F$
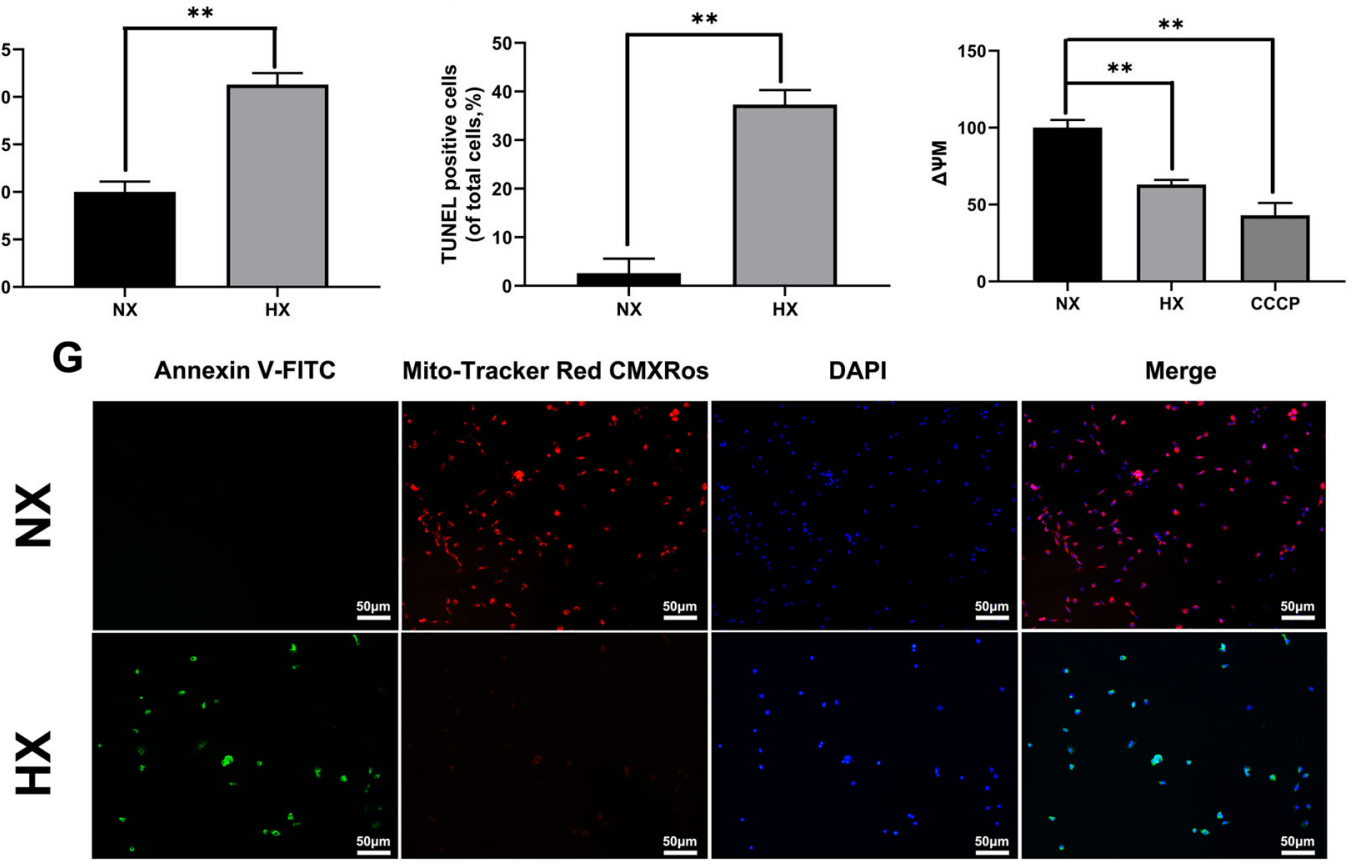

Fig. 2 Hypoxia stimulates mitochondrial ROS generation, apoptosis, and autophagy in chondrocytes. Chondrocytes stimulated with hypoxia condition $\left(1 \% \mathrm{pO}_{2}\right.$ ) for $24 \mathrm{~h}$. a The expression of LC3-I, II, P62, BCl-2, Bax and Cleaved-caspase3 from hypoxia treated chondrocytes were analyzed by western blot. b Quantification of LC3-I, II, P62, BCl-2, Bax and cleaved-caspase 3 by immunoblots. c-e Hypoxia induced mitochondria ROS and apoptosis in chondrocytes were analyzed by Mito-Sox stains and TUNEL assay. The fluorescence of Mito-Sox and TUNEL was quantified. $\mathbf{f} \triangle \boldsymbol{\Psi} M$ loss was measured by JC-1 and analyzed by flow cytometer. CCCP was used as positive control. $\mathbf{g}$ The Mito-tracker red probe was used to measure the mitochondrial membrane potential (MMP). Annexin V-FITC was used to assess apoptosis. All data represent mean \pm S.D. $(n=6)$. ${ }^{* *} P<0.01,{ }^{*} P<0.05$. 


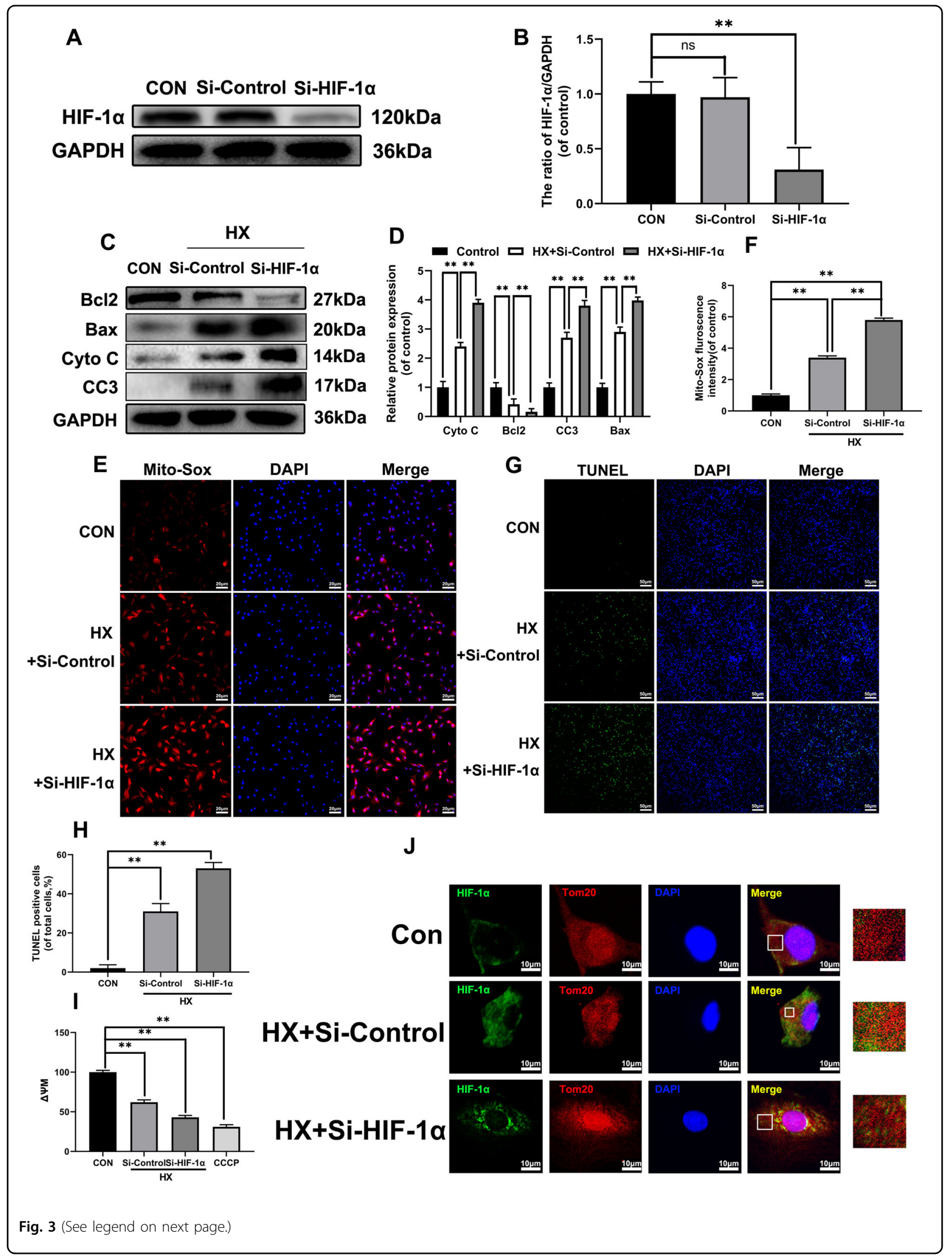


(see figure on previous page)

Fig. 3 HIF-1a is associated with hypoxia-induced chondrocytes self-protection. The chondrocytes were pretreated with HIF-1a target siRNA for $48 \mathrm{~h}$ followed hypoxia treatment $\left(1 \% \mathrm{pO}_{2}\right)$ for $24 \mathrm{~h}$. a The expression of HIF-1a was evaluated by western blots. b Quantification of HIF-1a immunoblots. c The expression of apoptotic proteins, including Bcl-2, Bax, Cyto C, and cleaved-caspase3 was evaluated by western blots. d Quantification of Bcl-2, Bax, Cyto C, and Cleaved-caspase 3 by immunoblots. e Mito-Sox was performed in chondrocytes. f The fluorescence intensity of Mito-sox in chondrocytes was quantified. $\mathbf{g}$ TUNEL assay was performed in chondrocytes. $\mathbf{h}$ The fluorescence intensity of TUNEL in chondrocytes was quantified. i JC-1 was measured in chondrocytes. $\mathbf{j}$ Representative image of immunofluorescence double staining of HIF-1a and Tom20 in chondrocytes. All data represent mean \pm S.D. $(n=6)$. ${ }^{* *} P<0.01,{ }^{*} P<0.05$.

DMOG promoted mitophagy and inhibited mitochondrial dysfunction, apoptosis through regulating HIF-1a expression in hypoxia stimulated chondrocytes

In Fig. 4, the results showed that DMOG could markedly activate autophagy and inhibit apoptosis. However, the potential effect of DOMG on autophagy was depending on HIF- $1 \alpha$ is unknown. Therefore, we knockdown the HIF- $1 \alpha$ by siRNA to ascertain the mechanism of DMOG on mitophagy. After pretreated with HIF- $1 \alpha$ siRNA, the ratio of LC3-II/LC3-I was sharply decreased, whereas the P62 expression was significantly increased in siRNA-HIF-1 $\alpha$ group. The BNIP3 expression level was up-regulated by DOMG treatment, while HIF-1 $\alpha$ knockdown effectively reduced the BNIP3 level relative to control (Fig. 5a, b). To determine whether DMOG could alleviate hypoxia induced mitochondrial dysfunction and apoptosis via increasing HIF-1 $\alpha$, we used western blot, TUNEL staining and Mito-Sox assay in vivo experiment. Bcl-2 expression levels were significantly decreased, while Bax and cleaved-caspase3 expression levels were remarkably increased in DMOG-treated chondrocytes with HIF-1 $\alpha$ siRNA (Fig. 5a, c). Co-localization of autophagosomes could be used for assessing the level of mitophagy mediated clearance of depolarized/dysfunctional mitochondria in hypoxia stimulated chondrocytes. Autophagosomes and mitochondria were stained with LC3 and Tom20 (mitochondrial outer membrane marker), respectively. By double immunofluorescence staining with LC3-II and Tom20, we observed that DMOG discernibly increased the LC3 positive autophagosomes formation which were co-localized with the mitochondria compared to the hypoxia group, which was reversed by siRNA- HIF-1 $\alpha$ (Fig. 5d). HIF-1 $\alpha$ siRNA remarkably reversed the increase of Tunnel positive cells and fluorescence intensity of Mito-Sox (Fig. 5e-g). In summary, DMOG significantly alleviated the mitochondrial dysfunction and apoptosis under hypoxia stimulation through HIF-1 $\alpha$-mediated mitophagy.

\section{DMOG regulated ECM-associated and senescence-related proteins and genes via HIF-1a}

The P16, P53, MMP-13 and ADAMTS5 genes expression level were measured by RT-qPCR to explore whether HIF- $1 \alpha$ participated in the cell senescence and ECM metabolism in chondrocytes. In addition, we detected the collagen II and aggrecan expressions by immunofluorescence. SA- $\beta$-galfor detecting senescent cells, and Edu assay for detecting the proliferation capacity were also assessed. As shown in Fig. 6d, the P16, P53, MMP-13 and ADAMTS5 protein expression levels were significantly enhanced in the hypoxia group compared to the other three groups. These genes were markedly reduced in chondrocytes with DMOG pretreatment for $2 \mathrm{~h}$ and another hypoxia induction for $24 \mathrm{~h}$. HIF- $\alpha$ knockdown significantly reduced the potential effects of DMOG. Furthermore, hypoxia could decrease both aggrecan and collagen II expression levels, which were partly reversed by DMOG administration. HIF- $1 \alpha$-siRNA group also showed less aggrecan and collagen II fluorescence intensity (Fig. 6a-c). For cell senescence and proliferation capacity, DMOG could markedly rescue aging cell from senescence induced by hypoxia stimulation, and improve proliferation capacity. Furthermore, this potential effect was reversed by knockdown of HIF-1 $\alpha$ (Fig. 6e-g). Thus, these important results indicated that DMOG could substantially inhibit the extracellular matrix degradation and cell aging through enhancing the HIF-1 $\alpha$.

\section{DMOG ameliorated OA development in DMM mouse model}

According to the vitro experiments, we investigated the potential effects of DMOG on imageology and histomorphology of knee joints in DMM mouse model. HIF-1 $\alpha$ immunohistochemical staining showed that DMOG could increase the HIF-1 $\alpha$ expression levels in vivo model (Fig. 7a, b). X ray imaging obtained at 8-week after surgery showed that DMM group exhibited aberrantly joint space, however, lower narrow of joint space was found in DMM +DMOG group (Fig. 7c). Erosion and hypo-cellularity of the superficial articular cartilage, and proteoglycan loss were observed in DMM group by Safranin O staining, HE staining and Alcian Blue staining at 8-week after surgery. In contrast, the DMM+DOMG group had more complete cartilage surface, and richer proteoglycan (Fig. 7d). Consistent with staining, OARSI score was declined by DMOG, and rising in DMM group (Fig. 7e). Meanwhile, in order to determine the effect of HIF- $1 \alpha$-mediated mitophagy on apoptosis in vivo, we detected the LC3II, 


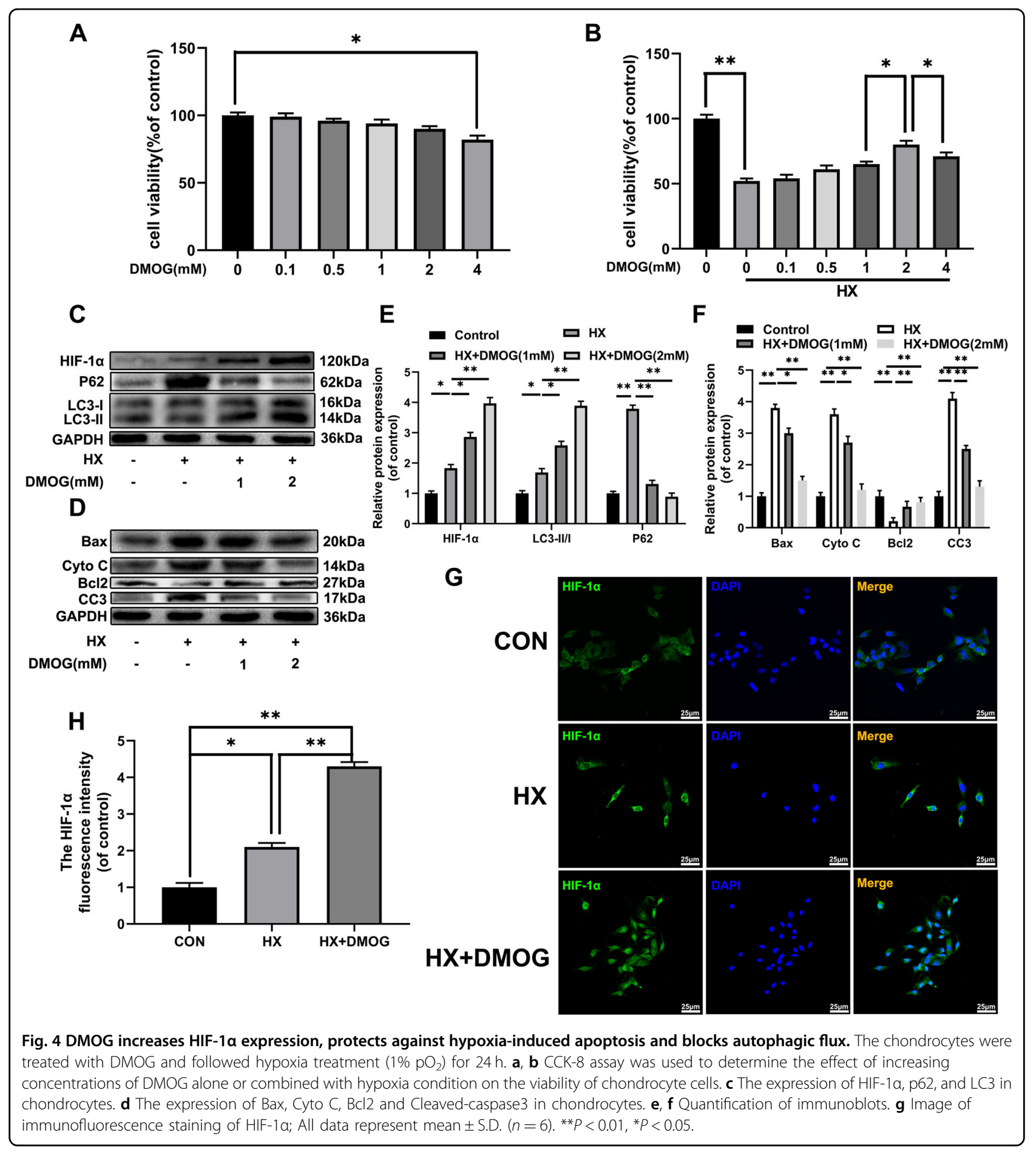

and Cleaved-caspase 3 expression levels by immunohistochemical staining. The results showed that increased DMOG induced HIF- $1 \alpha$ promoted LC3 expression and decreased Cleaved-caspase3 expression in chondrocytes (Fig. 7f, g). Therefore, these important findings revealed that DMOG-induced up-expression of HIF- $1 \alpha$ could alleviate osteoarthritis via activation of autophagy.

\section{Discussion}

OA is one of most prevalent musculoskeletal diseases and it is regarded as a central cause disability worldwide $^{31,32}$. OA leads to the articular cartilage degeneration and variation in subchondral bone ${ }^{33}$. In addition, it is accompanied by serious synovium inflammation and irreversible cartilage degradation, with the following 


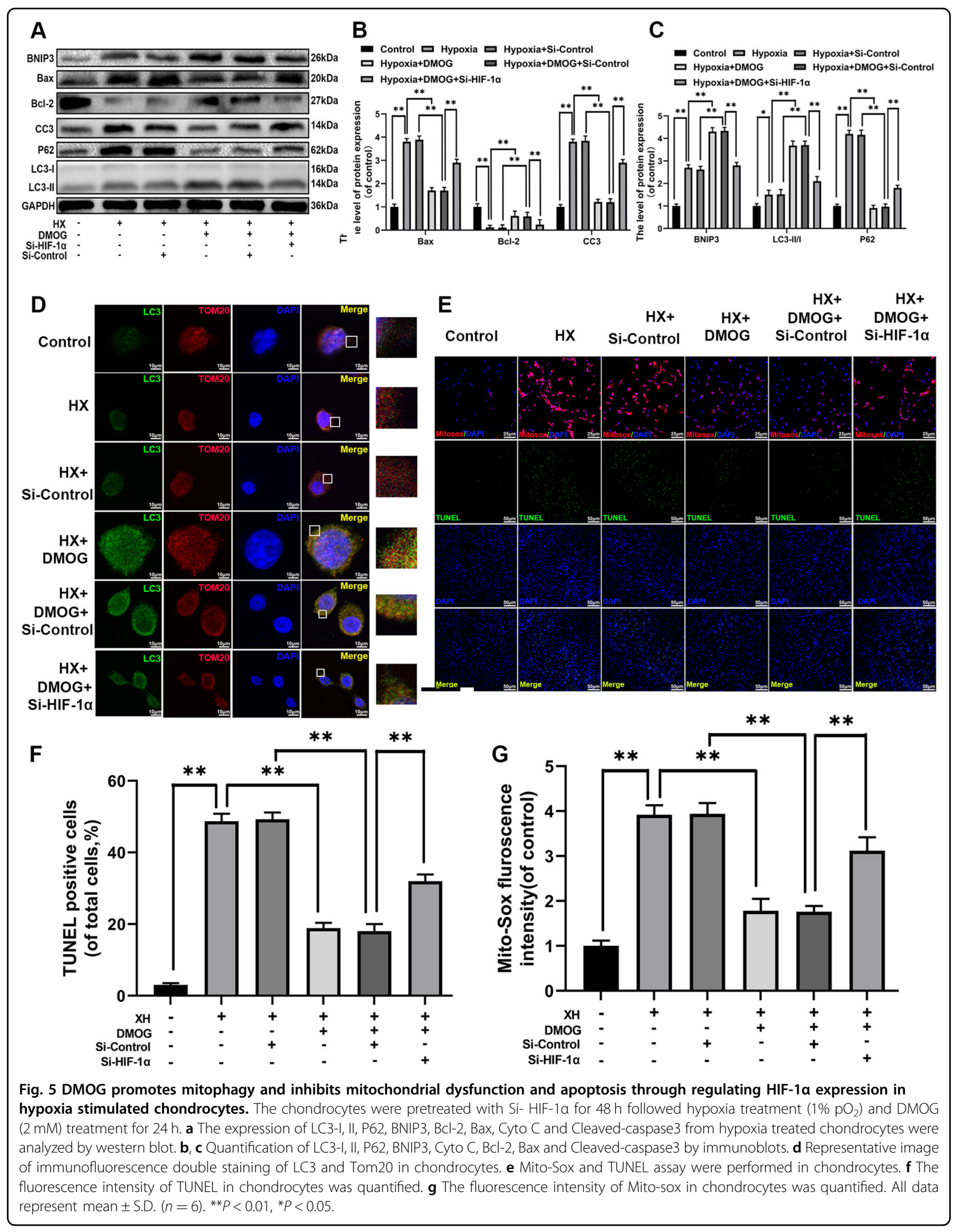



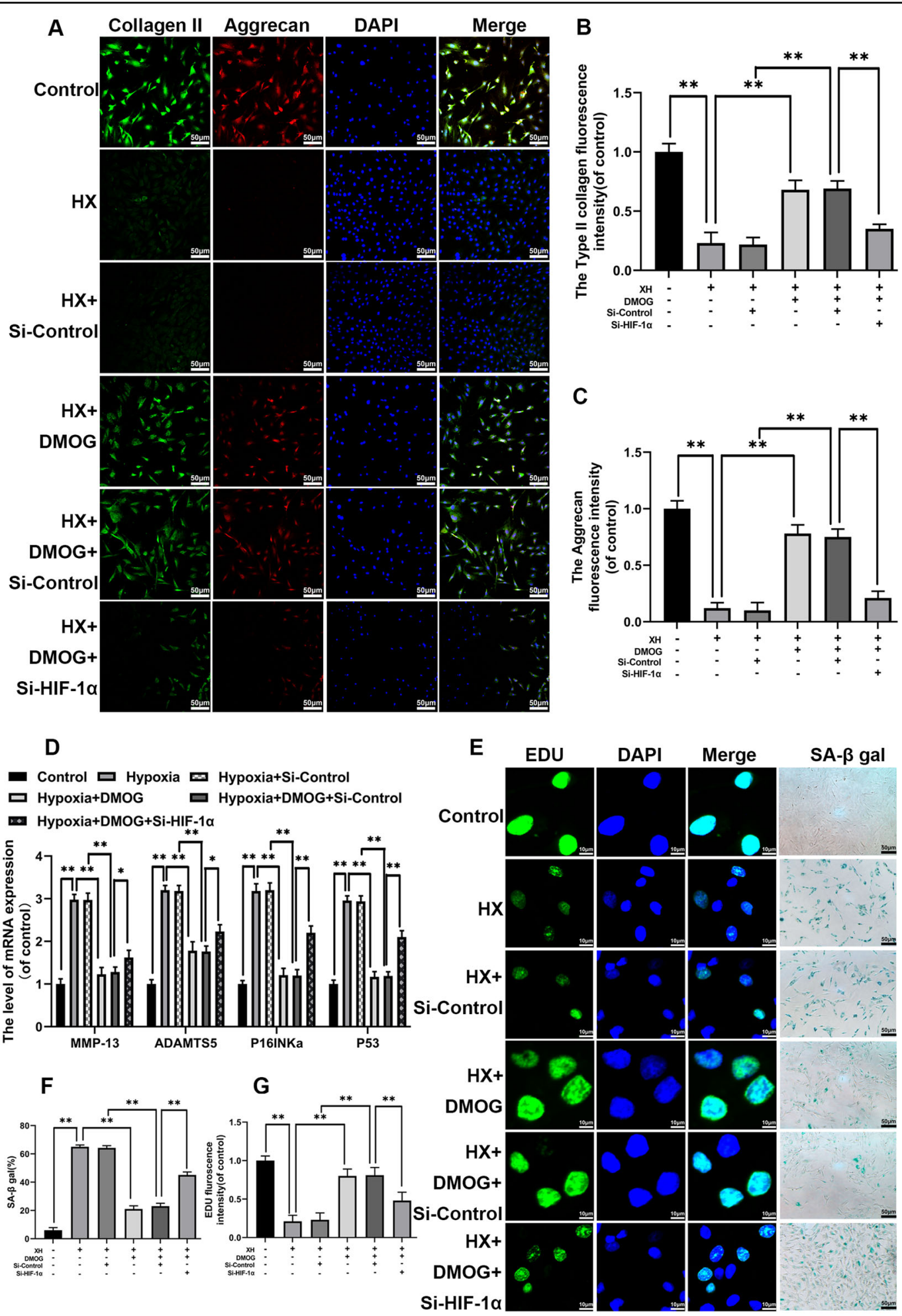

Fig. 6 DMOG regulates the expression of ECM-associated and senescence-related proteins and genes via promoting HIF-1a expression. The chondrocytes were pretreated with Si-HIF-1a for $48 \mathrm{~h}$ followed hypoxia treatment $\left(1 \% \mathrm{pO}_{2}\right)$ and DMOG $(2 \mathrm{mM})$ treatment for $24 \mathrm{~h}$. a The expression of Collagen II and Aggrecan were assessed by immunofluorescence. $\mathbf{b}, \mathbf{c}$ The fluorescence intensity analyzed using Image J. d The mRNA expression of MMP-13, ADAMTS5, P16 and P53 in chondrocytes treated as above. e- $\mathbf{g}$ EDU assay and SA- $\beta$ gal staining were performed in chondrocytes. All data represent mean \pm S.D. $(n=6) .{ }^{* *} P<0.01,{ }^{*} P<0.05$. 


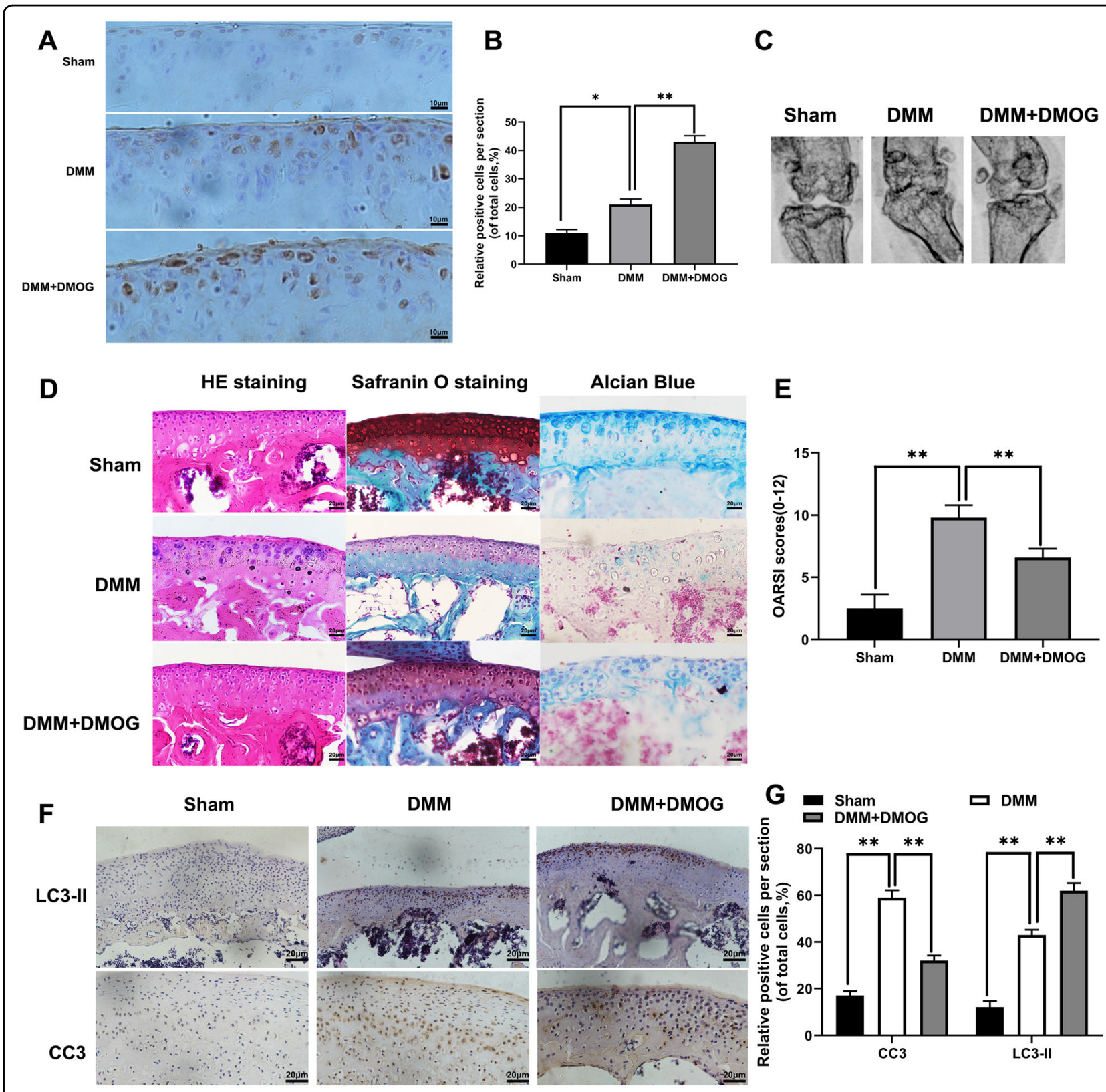

Fig. 7 DMOG ameliorates OA development in DMM mouse model. Osteoarthritis mouse model was established by surgical destabilization of the medial meniscus and 8-week injuryknee jointwere takenX-ray, RT-qPCR and stained with H\&E, Alcian Blue, Safranin Oandimmunohistochemistry staining. $\mathbf{a}, \mathbf{b}$ The expression of HIF-1a was evaluated by immunohistochemistry staining. c Digital X-ray image of mouse knee joints in different groups. $\mathbf{d}$ Representative HE, S-O, Alcian Blue staining of cartilage in each group at 8-week after surgery. $\mathbf{e}$ OARIS scores of cartilage in each group. $\mathbf{f}, \mathbf{g}$ The expression of LC3-II and Cleaved-caspase3 were evaluated by immunohistochemistry staining. All data represent mean $\pm S . D(n=6)$. ${ }^{* *} P<0.01$, ${ }^{*} P<0.05$.

major characteristics: progressive differentiation and maturity of the articular cartilage, progressive degradation of the extracellular matrix, progressive loss of the articular cartilage components ${ }^{34}$. From the perspective of cell morphology, a large number of apoptotic and senescent chondrocytes are the main cytological features of $\mathrm{OA}^{35,36}$. Extensive studies have documented that mitophagy leads to several diseases progression, including cancer $^{37}$, neurodegenerative diseases ${ }^{38}$ and cardiovascular disease ${ }^{39}$. Here, we sought to establish the potential role of HIF- $1 \alpha$-mediated mitophagy in eliminating dysfunctional mitochondria and its impact on remitting apoptosis and senescence in chondrocyte induced by hypoxia condition. 
HIF- $1 \alpha$ is a transcription factor acting as a physiological regulator, which maintains homeostasis in chondrocytes as well other cell types ${ }^{40,41}$. In normoxic conditions, prolyl hydroxylases (PHDs) hydroxylates HIF- $1 \alpha$ on proline residues (Pro402/564) in the oxygen-dependent degradation (ODD) domain. This hydroxylation process allows the HIF-1 $\alpha$ recognition by the von Hippel-Lindau (pVHL), a kind of tumor-suppressor protein and the substrate recognition component of an E3 ubiquitin ligase complex that aims HIF- $\alpha$ for proteasomal degrada$\operatorname{tion}^{42,43}$. On the contrary, under hypoxic conditions, HIF$1 \alpha$ hydroxylation is suppressed accumulating in the cytoplasm, allowing its phosphorylation and subsequent translocation to the nucleus, contributing to transcribing downstream target genes.

In current study, we found that HIF- $1 \alpha$ expression was notably increased in the OA human articular cartilage, mouse knee articular cartilage of DMM model and hypoxia treated rat chondrocytes. The dysregulated mRNAs identified in hypoxia treated rat chondrocytes by transcriptional analysis were enriched in several signaling pathways, including metabolic pathways, autophagy, apoptosis, senescence and the HIF-1 $\alpha$ signaling pathways. Furthermore, core mRNA BNIP3 was significantly enriched, a major target of HIF- $1 \alpha$ transcriptional genes and regarded as important mitophagy receptors and adaptors. Therefore, we hypothesized that HIF- $1 \alpha$ could alleviate hypoxia-induced apoptosis, senescence and matrix degradation in chondrocytes through mitophagy via enhancing BNIP3 expression. Although the protective effects of HIF$1 \alpha$ in osteoarthritis and rheumatoid arthritis in mice have been documented, there is no any clear data in this field showing the differential expression of HIF- $1 \alpha$ in the normal people and patients with osteoarthritis. As mentioned earlier, hypoxia leads to change in mitochondria, including decreased oxidative phosphorylation level, cytochrome c oxidase activity and increased ROS production. Among them, intracellular ROS are derived from mitochondria and membrane NADPH oxidase (Nox) system. Overinduced ROS is tightly related to age-related diseases, including $\mathrm{OA}$ and $\mathrm{IDD}^{44-46}$.

In our current study, the HIF- $1 \alpha$ expression was markedly elevated in the hypoxia-induced mice chondrocytes, together with apoptosis, cell senescence, ECM metabolic unbalance, and increased ROS and autophagy. Subsequent experiments showed that HIF- $1 \alpha$ regulated the eliminating hypoxia-induced cell damage and played an important role in ROS production. Interestingly, DMOG could increase HIF-1 $\alpha$ expression in both vitro and vivo, showing significant therapeutic effects against apoptosis and senescence. PHD is an important modulator of HIF- $1 \alpha$, could stabilize the HIF- $1 \alpha$ during hypoxia, indicating that PHD inhibitors may become potential candidates for intervening senescence and apoptosis ${ }^{47}$ To investigate the protective effect of HIF- $1 \alpha$ and the mechanism of its agonist DMOG, we performed the DMOG and HIF- $1 \alpha$ siRNA to regulate the HIF- $1 \alpha$ expression in chondrocytes. Our results suggested that the increased HIF- $1 \alpha$ expression could improve the chondrocytes survival $^{48,49}$.

Autophagy has been regarded as a protective mechanism under several pathological conditions through degrading useless proteins and organelles or dysfunctional organelles to maintain cellular functions ${ }^{50}$. In our current study, autophagy played an important role in preventing apoptosis and aging of chondrocytes to postpone OA. As a transcription factor and an oxygen sensor, phosphorylated HIF- $1 \alpha$ could hypoxia-mediated sensitive genes in nucleus. Among them, SOX9 and COL2A1 regulate chondrocyte function and maintain ECM anabolism. HIF1 could elevate type II collagen and Sox9 activity via activating hypoxia-induced transcriptional targeted genes $^{51,52}$. The stabilized HIF- $1 \alpha$ also could enhance the native type II collagen accumulation in the primary human chondrocytes, which could be inhibited by $2 \mathrm{ME} 2^{53}$. Therefore, these substantial findings suggested that HIF-1 $\alpha$ played an important role in maintaining the extracellular matrix integrity in articular cartilage. Furthermore, we found that HIF- $1 \alpha$ not only enhanced the extracellular matrix synthesis at the transcriptional level, but also inhibited the hypoxia-induced extracellular matrix degradation through enhancing mitophagy.

To explore the mechanism of HIF- $1 \alpha$ on mitophagy activation, we investigated the expression HiF-1 $\alpha /$ BNIP3 classical signaling pathway in the chondrocytes treated with DMOG under hypoxia. The BNIP3 expression is necessary for hypoxia-induced autophagy activation and BNIP3 knockdown aggravated cell death during hypoxia $^{54,55}$. In agreement with these previous studies, our study showed that the BNIP3 expression increased in a dose-dependent manner following the DMOG-induced HIF- $1 \alpha$ expression. The siRNA HIF- $1 \alpha$ could significantly reduce the BNIP3 expression and mitophagy, indicating that HIF-1 $\alpha /$ BNIP3 was a significant mechanism involved in DMOG protective effect.

HIF- $1 \alpha$ agonists, such as small molecule hypoxiainducing factor-prolyl hydroxylase inhibitor GSK1278863, used in clinical trials to treat anemia caused by chronic kidney disease without serious adverse effects $^{50}$. In addition, HIF- $1 \alpha$ has shown potential therapeutic effects in cancer and fracture repair. Therefore, HIF- $1 \alpha$ might be a potential target for alleviating OA.

We demonstrated that HIF-1 $\alpha$-mediated autophagy played a protective role in hypoxia-stimulated chondrocytes. However, precise mechanism on HIF- $1 \alpha$ inhibited apoptosis and senescence was unknown. The protective effect of HIF- $1 \alpha$ through inducing the transcription of anti-senescence and anti-apoptosis genes 
remained unclear. In the hypoxic environment, autophagy mechanism of reducing senescence and apoptosis needs to be further studied.

In conclusion, HIF- $1 \alpha$ was related to the OA progression, regulated mitochondrial dysfunctions via mitophagy in the damaged chondrocytes. DMOG could significantly inhibit apoptosis and chondrocytes senescence, and recover ECM metabolic unbalance in the chondrocytes via HIF- $1 \alpha$ mediated mitophagy.

\section{Materials and methods}

\section{Ethics statement}

All surgical interventions, treatments and postoperative animal care procedures were strictly performed accordance with the Animal Care and Use Committee of Wenzhou Medical University (ethic code: wydw20140129). The Human articular cartilage tissue collection and the experiments were approved by Ethical Committee of the Second Affiliated Hospital, Wenzhou Medical University and following the guidelines of the Declaration of Helsinki ${ }^{56}$

\section{Human cartilage and chondrocytes culture}

The normal human articular cartilages from 6 donors with no clinical symptoms and imaging features of OA were obtained from femoral condyles and tibial plateaus at autopsy (45-64 years old; mean, 53.8 years; Kellgren-Lawrence grade, 0 or I; $n=6$ ). The OA human articular cartilages were achieved from 6 patients (45-60 years old; mean, 56.1 years; Kellgren-Lawrence grade, III or IV; $n=6$ ) undergoing total knee arthroplasty. For the proposed activities, enrollment of specimen donors was performed by a strict procedure that has been approved by the Ethical Committee of the Second Affiliated Hospital, Wenzhou Medical University at participating institutions, with informed consent and the utmost attention to the issues of patient safety, anonymity and confidentiality. Human articular cartilages samples were cut into $1 \mathrm{~cm} 3$ pieces and incubated with $2 \mathrm{mg} / \mathrm{ml}$ of collagenase II in DMEM at $37^{\circ} \mathrm{C}$ for $4 \mathrm{~h}$. After washing by PBS for 3 times and resuspension, the chondrocytes from cartilage were cultured in cell culture dish at a seeding density of $3 \times 10^{5}$ cells per $\mathrm{ml}$ in DMEM/F12 contained with $10 \% \mathrm{FBS}$ and $1 \%$ antibiotic in $5 \% \mathrm{CO}_{2}$ at $37^{\circ} \mathrm{C}$. Cells from the second passage were used in subsequent experiments.

\section{Primary chondrocytes culture}

Twelve healthy C57BL/6 mice (6 males and 6 females, 5 days) were euthanized with sodium pentobarbital overdose. The cartilage of the knee joint of mice was carefully removed under microscope in a sterile environment. Then, the tissues were digested with $2 \mathrm{mg} / \mathrm{ml}$ (0.1\%) collagenase II for $4 \mathrm{~h}$ at $37^{\circ} \mathrm{C}$. Next, the digested tissue pieces were suspended and seeded into tissue culture flasks. The chondrocytes planted and expended in the condition of DMEM/F12 (Gibco, Invitrogen, Grand Island, NY) with $10 \%$ fetal bovine serum (FBS; Hyclone, Thermo Scientifc, Logan, UT, USA) and 1\% penicillin/ streptomycin antibiotics (Gibco, Invitrogen, Grand Island, $\mathrm{NY}$ ) in the incubator with $5 \% \mathrm{CO}_{2}$ at $37^{\circ} \mathrm{C}$. The medium was changed 3 times per week. Cultures were observed daily with an inverted phase contrast microscope. Cells from the second passage were suited for next experiments. All cells were tested negative for mycoplasma contamination.

\section{Hypoxic cell model}

Cells in good condition at logarithmic growth stage were selected, digested with $0.25 \%$ trypsin, centrifuged, supernatant removed, re-suspended and inoculated in cell culture plates for the corresponding experiment. The normal oxygen group was cultured in the cell incubator. The hypoxic group and the drug administration group were placed in the modular incubator chamber, and were given secondary vitality containing different concentrations of $\mathrm{N}_{2}$ and $\mathrm{CO}_{2}$. Experiments were carried out when the oxygen concentration in the incubator was maintained to the target concentration.

\section{RNA-sequencing and bioinformatics analysis}

Primary chondrocytes $\left(1 \times 10^{5}\right.$ cells per well $)$ were seeded in 6-well plates, grown to $80 \%$ confluence and treated with hypoxia condition $\left(1 \% \mathrm{pO}_{2}\right)$ for 1 day. Then, total RNA from cells of each group was extracted using TRIzol reagent (Invitrogen) according to the manufacturer's instructions. RNA-sequencing and bioinformatics analyses were performed as previously described ${ }^{57}$.

\section{Si-RNA transfections}

Effective siRNAs for HIF- $1 \alpha$ and control siRNA were obtained from Invitrogen (Carlsbad, CA, USA). When reaching $50-60 \%$ confluence cells, chondrocytes were transfected with HIF-1 $\alpha$ siRNA using Lipofectamine 2000 siRNA transfection reagent (Thermo Fisher, UT, USA) according to the manufacturer's protocols.

\section{Cell viability assay}

Cell viability was detected using CCK8 according to the manufacturer's instructions. Briefly, chondrocytes were planted into the 96 well-plate (8000 cells/well) and incubated in DMEM/F12 with $10 \%$ FBS at $37^{\circ} \mathrm{C}$ for $24 \mathrm{~h}$. Then, chondrocytes were treated with the different concentration of DMOG as described above. A total of $10 \mu \mathrm{L}$ CCK-8 solution was added into each well. And the plate was incubated for an additional $1 \mathrm{~h}$. The absorbance of samples was measured at $450 \mathrm{~nm}$ using a microplate reader (Thermo Scientific, USA). 


\section{Western blot analysis}

After isolating total protein from chondrocytes using RIPA buffer with $1 \mathrm{mM}$ phenylmethanesulfonylfluoride (PMSF), the protein concentration was measured by BCA protein assay kit (Beyotime). Protein samples were separated by sodium dodecyl sulfatepolyacrylamide gel electrophoresis (SDS-PAGE) and then transferred to a $0.45 \mu \mathrm{m}$ polyvinylidene difluoride membranes (Millipore, USA). Following blocking with 5\% non-fat milk for $2 \mathrm{~h}$, the membranes were incubated with primary antibodies against HIF- $1 \alpha$ (1:500), cleaved-caspase3 (1:1000), Bax (1:1000), Bcl-2 (1:1000), Cyto C (1:1000), LC3 (1:1000), p62 (1:1000), GAPDH (1:1000), and BNIP3 (1:500) overnight at $4{ }^{\circ} \mathrm{C}$. Then, bands were incubated with the respective secondary antibodies. Finally, the bands were detected using electrochemiluminescence plus reagent (Invitrogen). Image Lab 3.0 software (Bio-Rad) was used to quantified the intensity of protein bands.

\section{Real-time PCR}

The total RNA was extracted using TRIzol reagent (Invitrogen, Grand Island, NY). One microgram of total RNA was used to synthesize cDNA (MBI Fermantas, Germany). For quantitative real-time PCR (qPCR), a total $10 \mu \mathrm{l}$ of reaction volume, including $5 \mu \mathrm{l}$ of $2 \times$ SYBR Master Mix, $0.25 \mu \mathrm{l}$ of each primer and $4.5 \mu \mathrm{l}$ of diluted cDNA was used. The machine parameters of RT-PCR were: $10 \mathrm{~min} 95^{\circ} \mathrm{C}$ followed by 40 cycles of $15 \mathrm{~s} 95^{\circ} \mathrm{C}$ and $1 \mathrm{~min} 60^{\circ} \mathrm{C}$. The reaction was performed using CFX96Real-Time PCR System (Bio-Rad Laboratories, California, USA). The cycle threshold $(\mathrm{Ct})$ values were collected and normalized to the GAPDH level. The results were assessed by using the $2-\Delta \Delta \mathrm{Ct}$ method. The primer sequences were as follow: for MMP-13, Forward $5^{\prime}-\mathrm{G}$ TGATGATGATGATGATGAC-3', Reverse 5'-GCAGGA TGG TAGTATGATT-3'; for ADAMTS5, Forward 5'-T CTCCAAAGGTTACGGATGGGR-3', Reverse $5^{\prime}$-TCTT CTTCAGGGCTAAGTAGGCAG-3'; for P16, Forward 5'-CTCCTGAAAATCAAGGGTTGAG-3', Reverse $3^{\prime}$-A CCTTCCTAACTGCCAAATTGA-5'; for P53, Forward 5'-GTGAGGGATGTTTGGGAGATG-3', Reverse 5' - CC TGGTTAGTACGGTGAAGTG-3'.

\section{Immunohistochemistry}

After double deparaffinization and hydration through xylene and graded alcohol series, sample sections were incubated with $3 \% \mathrm{H}_{2} \mathrm{O}_{2}$ for $10 \mathrm{~min}$ and washed by PBS for 3 times. Then, the tissue sections were incubated with $0.1 \%$ trypsin for $20 \mathrm{~min}$ and washed by PBS for 3 times. After blocking with $10 \%(\mathrm{w} / \mathrm{v}) \mathrm{BSA}$ for $1 \mathrm{~h}$ at $37^{\circ} \mathrm{C}$, the sections were incubated with specific primary HIF- $1 \alpha$, LC3II, Cleaved-caspase3 antibody (Abcam, USA, 1:200) at $4{ }^{\circ} \mathrm{C}$ overnight. Negative control sections were incubated with non-specific IgG. After incubation at $37^{\circ} \mathrm{C}$ for $1 \mathrm{~h}$, the tissue sections were washed by PBS for 3 times and incubated with HRP-conjugated secondary antibodies for $1 \mathrm{~h}$ at $37^{\circ} \mathrm{C}$. Color development was performed using a DAB color development kit (ZhongShan Biotech). At least 3 sections from each specimen were observed. The rate of positive cells each section was measured by observers who were blinded to the experimental groups. The positive points were quantified by Image J software 2.1 (Bethesda, MD, USA).

\section{Immunofluorescence}

Cells were fixed on coverslips, and blocked by $10 \%$ goat serum for $1 \mathrm{~h}$ at $37^{\circ} \mathrm{C}$. Samples were then probed using primary antibodies HIF-1 $\alpha$ (1:100, Abcam), Tom20 (1:100, Santa Cruz Biotechnology), rabbit anti-LC3 (1:100, Cell Signaling Technology), Collagen type II (1:100, Abcam) and Aggrecan (Santa Cruz Biotechnology, 1:50) at $4{ }^{\circ} \mathrm{C}$ overnight. Then slides were incubated with fluorescein isothiocyanate or tetramethyl rhodamine isothiocyanate conjugated second antibodies for $45 \mathrm{~min}$ and labeled with 40, 6-diamidino-2-phenylindole (DAPI, Beyotime) for $3 \mathrm{~min}$. finally, slides were assessed in a confocal fluorescence microscope (Nikon, Japan). In addition, fluorescence intensity was measured using Image J software 2.1 (Bethesda, MD, USA).

\section{Measurement of mitochondrial Reactive oxygen species (ROS)}

Mitochondrial ROS was measured by using Mito-SOX Red dye (Invitrogen, M36008). NP cells were plated in sixwell plates. After treated above, cells were incubated with Mito-Sox Red $(5 \mu \mathrm{M})$ for $30 \mathrm{~min}$ at $37^{\circ} \mathrm{C}$, then washed by PBS for 2 times, and observed by the confocal fluorescence microscope (Nikon, Japan).

\section{Mitochondrial membrane potential ( $\Delta \Psi \mathrm{M})$}

$\Delta \Psi_{\mathrm{M}}$ was assessed using the mitochondrial-specific fluorescent probe JC-1 (5, 5', 6, 6'-124 tetrachloro-1, $1^{\prime}, 3$, $3^{\prime}$ - tetraethyl-benzimidazolylcarbocyanine iodide) (Yeasen Biochemical, Shanghai, China). Brief, cartilage cells were incubated with JC-1 $(5 \mu \mathrm{M})$ for $30 \mathrm{~min}$ at $37^{\circ} \mathrm{C}$ and washed by PBS for 3 times and observed by the Flow cytometer.

\section{Mito-tracker red staining}

Mito-tracker red CMXRos (Yeasen Biochemical, Shanghai, China) was used to determine the mitochondria level in live cells. According to the manufacturer's instructions, cartilage cells were incubated with Mitotracker probes at the $50 \mathrm{nM}$ concentration for $30 \mathrm{~min}$ at $37^{\circ} \mathrm{C}$. Then, cell nuclei were stained with DAPI for $5 \mathrm{~min}$ at $37^{\circ} \mathrm{C}$. NP cells were washed by PBS for 3 times and imaged with the confocal fluorescence microscope (Nikon, Japan). The fluorescence intensity was quantified by using Image J software 2.1 (Bethesda, MD, USA). 


\section{SA- $\beta$-gal staining}

Senescence level was measured by senescence associated $\beta$-galactosidase (SA- $\beta$-gal) staining kit (Beyotime, Shanghai, China) according to the manufacturer's instructions. Aging chondrocytes showing higher SA$\beta$-gal activity were stained blue.

\section{TUNEL staining}

TUNEL staining was used to detect the damaged DNA level. Chondrocytes were fixed and then stained with in situ cell death detection kit (Yeasen Biochemical, Shanghai, China) according to the manufacturer's instructions for $30 \mathrm{~min}$ at $37^{\circ} \mathrm{C}$ and the nuclei was stained with DAPI. Twelve fields of each slide were randomly selected and captured under Nikon ECLIPSE Ti microscope to count TUNEL positive cells.

\section{Animal model}

Sixty 10-week-old C57BL/6 mice (30 male and 30 female) were purchased from Animal Center of Chinese Academy of Sciences (Shanghai, China) and were divided randomly into the sham group, DMM group and DMM +DMOG group. OA mouse model was established by surgical destabilization of the medial meniscus as previously described ${ }^{58}$. For DMM+DMOG group, DMOG was administrated to animals by intraperitoneal (i.p.) injection at $25 \mathrm{mg} / \mathrm{kg}$ per day. Mice were sacrificed at 8 weeks post-OA surgery from each group. The knee joints were dissected and processed for histological evaluation. The investigator was blinded to the group allocation during the experiment and when assessing the outcome.

\section{X-ray imaging method}

After 8 weeks of surgery, the animals were given the $\mathrm{X}$-ray examination. $\mathrm{X}$-ray imaging was performed on all mice to evaluate the joint space using a digital X-ray machine (Kubtec Model XPERT.8; KUB Technologies). Proper images were obtained in the following settings: $50 \mathrm{Kv}$ and $160 \mu \mathrm{A}$.

\section{Histopathological analysis}

The mice were sacrificed by $10 \%$ chloral hydrate intraperitoneal injection and the knee joint were harvested 8 weeks after surgery. The specimens were fixed in $4 \%$ $(\mathrm{v} / \mathrm{v})$ paraformaldehyde for $24 \mathrm{~h}$ and decalcified in $10 \%$ (v/v) ETDA. After dehydrated, tissues were embedded in paraffin and cut into $5-\mu \mathrm{m}$ sections. The sample slides were stained with $\mathrm{HE}$ staining, safranin $\mathrm{O}$-fast green (S-O) and Alcian Blue. Mice in each group were evaluated by Osteoarthritis Research Society International (OARSI) scoring system for medial femoral condyle and medial tibial plateau.

\section{Statistical analysis}

The results were expressed as mean \pm S.D. Raw statistical analyses were processed by using Graphpad Prism 6 (USA). Nonparametric data were analyzed by Mann-Whitney $U$-test. Data were analyzed by one-way analysis of variance (ANOVA) followed by the Tukey's test for comparison between different groups. All the experiments were performed in six times and were consistently repeatable.

\section{Acknowledgements \\ This work is supported by grants from National Nature Foundation of China (81722028, 81871806, 81972150), Zhejiang Public Service Technology Research Program/Social Development (LGF18H060008). \\ Author details \\ 'Department of Orthopaedics, The Second Affiliated Hospital and Yuying Children's Hospital of Wenzhou Medical University, Wenzhou, Zhejiang, China. ${ }^{2}$ Molecular Pharmacology Research Center, School of Pharmaceutical Science, Wenzhou Medical University, Wenzhou, Zhejiang, China. ${ }^{3}$ The Second School of Medicine, Wenzhou Medical University, Wenzhou, Zhejiang, China. \\ ${ }^{4}$ Department of Orthopaedics, The first Affiliated Hospital of Wenzhou Medical University, Wenzhou, Zhejiang, China}

Conflict of interest

The authors declare that they have no conflict of interest.

\section{Publisher's note}

Springer Nature remains neutral with regard to jurisdictional claims in published maps and institutional affiliations.

Supplementary Information accompanies this paper at (https://doi.org/ 10.1038/s41419-020-2680-0).

Received: 9 February 2020 Revised: 4 June 2020 Accepted: 8 June 2020 Published online: 25 June 2020

References

1. Daniel, P.-A. et al. Incidence and risk factors for clinically diagnosed knee, hip and hand osteoarthritis: influences of age, gender and osteoarthritis affecting other joints. Ann. Rheum. Dis. 73, 1659-1664 (2014).

2. Hunter, D. J., Schofield, D. \& Callander, E. The individual and socioeconomic impact of osteoarthritis.Nat. Rev. Rheumatol. 10, 437-441 (2014).

3. Yu, D., Peat, G., Bedson, J. \& Jordan, K. P. Annual consultation incidence of osteoarthritis estimated from population-based health care data in England. Rheumatology 54, 2051-2060 (2015).

4. Turkiewicz, A. et al. Current and future impact of osteoarthritis on health care: a population-based study with projections to year 2032. Osteoarthritis Cartilage 22, 1826-1832 (2014).

5. Neogi, T. The epidemiology and impact of pain in osteoarthritis. Osteoarthr. Cartil. 21, 1145-1153 (2013).

6. Block, J. A. Osteoarthritis: OA guidelines: improving care or merely codifying practice? Nat. Rev. Rheumatol. 10, 324-326 (2014).

7. Hamacher-Brady, A. \& Brady, N. R. Mitophagy programs: mechanisms and physiological implications of mitochondrial targeting by autophagy. Cell Mol. Life Sci. 73, 775-795 (2016).

8. Blanco, F. J., Lopez-Armada, M. J. \& Maneiro, E. Mitochondrial dysfunction in osteoarthritis. Mitochondrion 4, 715-728 (2004).

9. Jaiswal, $\mathrm{N}$. et al. Fructose induces mitochondrial dysfunction and triggers apoptosis in skeletal muscle cells by provoking oxidative stress. Apoptosis $\mathbf{2 0}$ 930-947 (2015).

10. Carames, B. et al. Autophagy is a protective mechanism in normal cartilage and its aging-related loss is linked with cell death and osteoarthritis. Arthritis Rheum. 62, 791-801 (2010). 
11. Caramés, B., Olmer, M., Kiosses, W. B. \& Lotz, M. K. The relationship of autophagy defects to cartilage damage during joint aging in a mouse model. Arthritis Rheumatol. 67, 1568-1576 (2015).

12. Ribeiro, M. et al. Diabetes-accelerated experimental osteoarthritis is prevented by autophagy activation. Osteoarthritis. Cartilage 24, 2116-2125 (2016).

13. Carames, B. et al. Autophagy activation by rapamycin reduces severity of experimental osteoarthritis. Ann. Rheum. Dis. 71, 575-581 (2012).

14. Dolman, N. J. et al. Tools and techniques to measure mitophagy using fluorescence microscopy. Autophagy 9, 1653-1662 (2013).

15. Kubli, D. A. \& Gustafsson, A. B. Mitochondria and mitophagy: the yin and yang of cell death control. Circ. Res. 111, 1208-1221 (2012).

16. Matsumoto, S. et al. Msp1 clears mistargeted proteins by facilitating their transfer from mitochondria to the ER. Mol. Cell 76, 191-205 (2019).

17. Lou, G. et al. Mitophagy and neuroprotection. Trends Mol. Med. 26, 8-20 (2019).

18. Livingston, M. J. et al. Clearance of damaged mitochondria via mitophagy is important to the protective effect of ischemic preconditioning in kidneys. Autophagy 15, 1-21 (2019)

19. Trollmann, R. et al. Pharmacologic stabilization of hypoxia-inducible transcription factors protects developing mouse brain from hypoxia-induced apoptotic cell death. Neuroscience 278, 327-342 (2014)

20. Schafer, $M$. et al. Role of redox signaling in the autonomous proliferative response of endothelial cells to hypoxia. Circ. Res. 92, 1010-1015 (2003).

21. Semenza, G. L. Oxygen sensing, hypoxia-inducible factors, and disease pathophysiology. Annu. Rev. Pathol. 9, 47-71 (2014).

22. Kondylis, V. \& Pasparakis, M. RIP kinases in liver cell death, inflammation and cancer. Trends Mol. Med. 25, 47-63 (2019).

23. Lampert, M. A. et al. BNIP3L/NIX and FUNDC1-mediated mitophagy is required for mitochondrial network remodeling during cardiac progenitor cell differentiation. Autophagy 15, 1182-1198 (2019).

24. Ney, P. A. Mitochondrial autophagy: origins, significance, and role of BNIP3 and NIX. Biochim. Biophys. Acta 1853, 2775-2783 (2015).

25. Taylor, C. T. Mitochondria and cellular oxygen sensing in the HIF pathway. Biochem. J. 409, 19-26 (2008).

26. Charlier, E. et al. Insights on molecular mechanisms of chondrocytes death in osteoarthritis. Int. J. Mol. Sci. 17, https://doi.org/10.3390/ijms17122146 (2016).

27. Schipani, E. et al. Hypoxia in cartilage: HIF-1alpha is essential for chondrocyte growth arrest and survival. Genes Dev. 15, 2865-2876 (2001).

28. Bohensky, J. et al. HIF-1 regulation of chondrocyte apoptosis: induction of the autophagic pathway. Autophagy 3, 207-214 (2007).

29. Bohensky, J., Leshinsky, S., Srinivas, V. \& Shapiro, I. M. Chondrocyte autophagy is stimulated by HIF-1 dependent AMPK activation and mTOR suppression. Pediatr. Nephrol. 25, 633-642 (2010).

30. Chen, G. et al. Hypoxia-induced microRNA-146a represses Bcl-2 through Traf6/ IRAK1 but not Smad4 to promote chondrocyte autophagy. Biol. Chem. 398, 499-507 (2017).

31. Jeffries, M. A. Osteoarthritis year in review 2018: genetics and epigenetics. Osteoarthritis Cartilage 27, 371-377 (2019).

32. Mandl, L. A. Osteoarthritis year in review 2018: clinical. Osteoarthritis Cartilage 27, 359-364 (2019).

33. Thysen, S., Luyten, F. P. \& Lories, R. J. U. Targets, models and challenges in osteoarthritis research. Dis. Model Mech. 8, 17-30 (2015).

34. Xu, Y. S. et al. Analysis of differences in the molecular mechanism of rheumatoid arthritis and osteoarthritis based on integration of gene expression profiles. Immunol. Lett. 168, 246-253 (2015).

35. Szychlinska, M. A., Ravalli, S. \& Musumeci, G. Pleiotropic effect of fibrates on senescence and autophagy in osteoarthritis. EBioMedicine 45, 11-12 (2019).
36. Loeser, R. F. Aging and osteoarthritis: the role of chondrocyte senescence and aging changes in the cartilage matrix. Osteoarthritis Cartilage 17, 971-979 (2009).

37. de Castro, G. S. et al. Human cachexia induces changes in mitochondria autophagy and apoptosis in the skeletal muscle. Cancers 11, https://doi.org/ 10.3390/cancers11091264 (2019).

38. Sardoiwala, M. N. et al. Recuperative effect of metformin loaded polydopamine nanoformulation promoting EZH2 mediated proteasomal degradation of phospho-a-Synuclein in Parkinson's disease model. Nanomedicine, 102088, https://doi.org/10.1016/j.nano.2019.102088 (2019).

39. Bravo-San Pedro, J. M., Kroemer, G. \& Galluzzi, L. Autophagy and mitophagy in cardiovascular disease. Circ. Res. 120, 1812-1824 (2017).

40. Schipani, E. et al. Hypoxia in cartilage: HIF-1alpha is essential for chondrocyte growth arrest and survival. Genes Dev. 15, 2865-2876 (2001).

41. Lee, H. J. et al. BICD1 mediates HIF1a nuclear translocation in mesenchymal stem cells during hypoxia adaptation. Cell Death Differ. 26, 1716-1734 (2019).

42. Patel, S. A. \& Simon, M. C. Biology of hypoxia-inducible factor-2alpha in development and disease. Cell Death Differ. 15, 628-634 (2008).

43. Findlay, D. M. \& Atkins, G. J. Osteoblast-chondrocyte interactions in osteoarthritis. Curr. Osteoporos. Rep. 12, 127-134 (2014).

44. Lepetsos, P. \& Papavassiliou, A. G. ROS/oxidative stress signaling in osteoarthritis. Biochim. Biophys. Acta 1862, 576-591 (2016).

45. Chang, S. H. et al. Excessive mechanical loading promotes osteoarthritis through the gremlin-1-NF-kB pathway. Nat. Commun. 10, 1442 (2019).

46. Yang, L. et al. Pyrroloquinoline quinone protects nucleus pulposus cells from hydrogen peroxide-induced apoptosis by inhibiting the mitochondriamediated pathway. Eur. Spine J. 24, 1702-1710 (2015).

47. Epstein, A. C. et al. C. elegans EGL-9 and mammalian homologs define a family of dioxygenases that regulate HIF by prolyl hydroxylation. Cell 107, 43-54 (2001).

48. Kong, L. et al. Bioactive injectable hydrogels containing desferrioxamine and bioglass for diabetic wound healing. ACS Appl. Mater. Interfaces 10, 30103-30114 (2018)

49. Montemurro, C. et al. IAPP toxicity activates HIF1a/PFKFB3 signaling delaying $\beta$-cell loss at the expense of $\beta$-cell function. Nat. Commun. 10, 2679 (2019).

50. Dutta, D. et al. Upregulated autophagy protects cardiomyocytes from oxidative stress-induced toxicity. Autophagy 9, 328-344 (2013).

51. Zhang, $C$. et al. Hypoxia-inducible factor- 1 is a positive regulator of Sox 9 activity in femoral head osteonecrosis. Bone 48, 507-513 (2011).

52. Grimmer, C. et al. Regulation of type II collagen synthesis during osteoarthritis by prolyl-4-hydroxylases: possible influence of low oxygen levels. Am. J. Pathol. 169, 491-502 (2006)

53. Gelse, K. et al. Role of hypoxia-inducible factor 1 alpha in the integrity of articular cartilage in murine knee joints. Arthritis Res. Ther. 10, R111 (2008).

54. Bellot, G. et al. Hypoxia-induced autophagy is mediated through hypoxiainducible factor induction of BNIP3 and BNIP3L via their BH3 domains. Mol. Cell. Biol. 29, 2570-2581 (2009).

55. Kang, C. et al. 6-Gingerols (6G) reduces hypoxia-induced PC-12 cells apoptosis and autophagy through regulation of miR-103/BNIP3. Artif. Cells Nanomed. Biotechnol. 47, 1653-1661 (2019).

56. World Medical Association, World Medical Association Declaration of Helsinki: ethical principles for medical research involving human subjects. JAMA $\mathbf{3 1 0}$ 2191-2194 (2014)

57. Zhang, S. et al. Core regulatory RNA molecules identified in articular cartilage stem/progenitor cells during osteoarthritis progression. Epigenomics 11 669-684 (2019).

58. Glasson, S. S., Blanchet, T. J. \& Morris, E. A. The surgical destabilization of the medial meniscus (DMM) model of osteoarthritis in the 129/SvEv mouse. Osteoarthritis Cartilage 15, 1061-1069 (2007). 